\title{
DETERMINATION OF SOIL MOISTURE BY NEUTRON SCATTERING
}

\author{
P. MORTIER and M. DE BOODT
}

Rijkslandbouwhogeschool, Gent, Belgium

1 The pioneer work concerning soil moisture determination by the method of neutron scattering has been carried out by W. Gardner and D. Kirkham (1952) and by D. J. Belcher (1950, 1953). Similar measurements have been done by other investigators.

The principle of the method is well known. In a given medium fast neutrons emitted e.g. by a Ra-Be source are first slowed down to neutrons with thermal energy, after covering an average slowing down length $l$. The thermal neutron diffuses until it disappears by capture. Putting the distance covered during this process equal to $6 L$, then $L$ is the diffusion length. According to the theory put forward by Wallace and afterwards by W. GardnER and D. KirkHAM (1952) the density $\varrho$ of thermal neutrons in the vicinity of a point source is a function of $r$, distance to the source, and $\gamma=l / L$.

At sufficiently small values of $r, \varrho$ is shown by these authors to increase rapidly with decreasing $\gamma$.

With increasing hydrogen content of the medium, $l$ decreases rapidly owing to the energy losses suffered by a fast neutron through collisions with $\mathrm{H}$ nuclei. $L$ may be assumed to remain relatively constant in working conditions encountered in soils (GARDNER and KIRKHAM, 1952).

Under these circumstances $\gamma$ is seen to decrease. As a consequence the density $\varrho$, measured at a small, constant distance from the source, determines the total hydrogen content of the medium. As in soils hydrogen is present essentially under the form of moisture, $\varrho$ measures in fact the moisture content.

2 The calibration curves of Fig. 1 were drawn in the following conditions. Samples of clay, loam and sand, with moisture contents determined by dessication at $105^{\circ} \mathrm{C}$, were put in a large cylindrical container. In the center of the sample was put a 1000 mc Ra-Be source, surrounded with a small rigid indium cylinder acting as detector of slow neutrons. It is activated according to the process ${ }_{115}^{49}$ In $(n, \gamma)_{116^{49}}^{4 n}$, the radio-active isotope decaying with a period of 54 minutes, under the emission of $\beta$ particles. The activity $N$ of the detector (counts per unit time), determined after a suitable irradiation time (in practice 30 minutes), is a measure of the moisture content.

We obtained one calibration curve for clay and another one for loam and sand. For moisture contents above $100 \mathrm{gr} / \mathrm{dm}^{3}$ the accuracy obtainable by the radio-active method is better than $10 \mathrm{gr} / \mathrm{dm}^{3}$. It is somewhat poorer for smaller moisture contents.

The shift of the calibration curve for clay against the one for loam is probably significant. It is reasonably constant and corresponds, for a given $N$, to a surplus of total water content of about $10 \mathrm{gr} / \mathrm{dm}^{3}$. We think this increase 


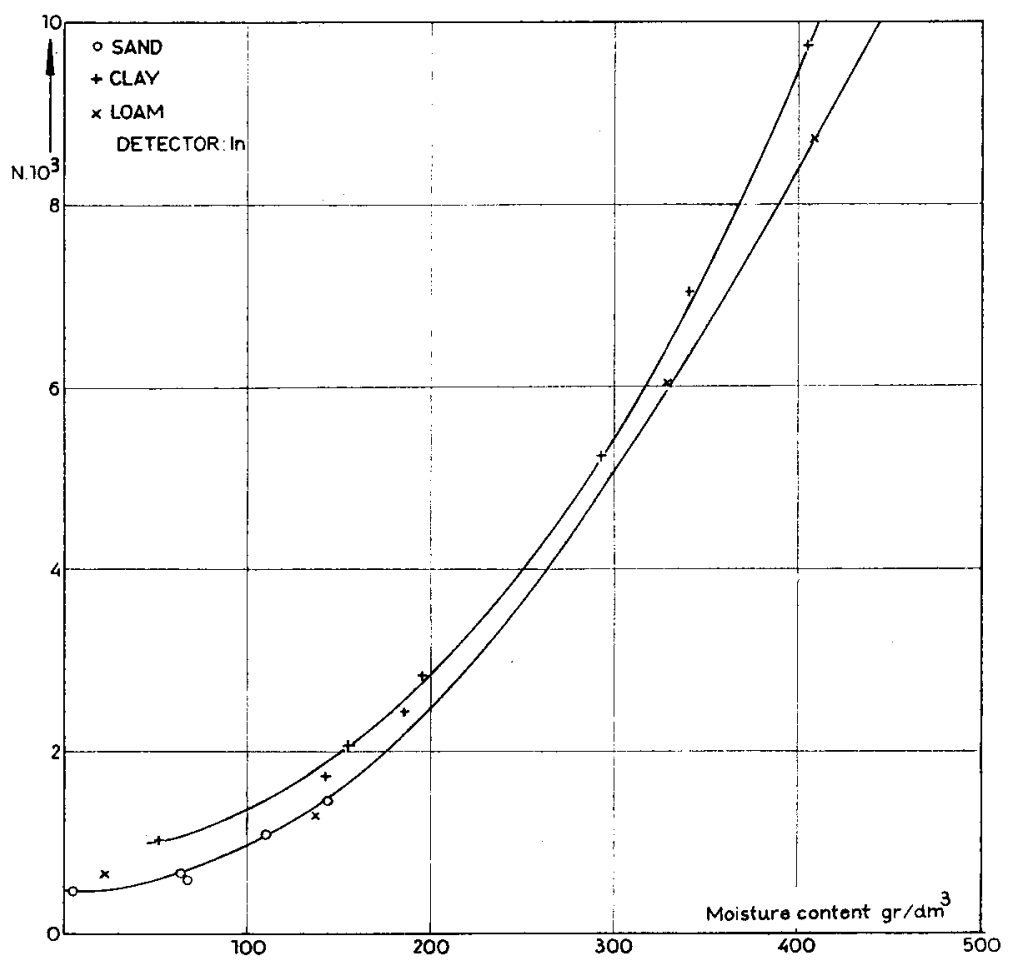

Fic. 1. Counting rate vs. moisture content for several soils.

should be attributed to the difference of the amount of crystallization and imbitition water above $105^{\circ} \mathrm{C}$ present in the two soils. The granulometric analysis of the soil samples showed the $0-2 \mathrm{mu}$ fraction to be $27.3 \%$ for clay and $12.2 \%$ for loam.

From previous work it is known that for soils, above $105^{\circ} \mathrm{C}$, practically all the bound water is contained in this fraction. The amount of the bound water for soils containing hydromicas, as it is the fact in our case, was found to be $6 \%$ by weight of the $0-2$ mu fraction (DE Leenhere, 1941). From the known apparent densities of the samples, the amount of water bound above $105^{\circ} \mathrm{C}$ was found to be $17 \mathrm{gr} / \mathrm{dm}^{3}$ for clay and $7 \mathrm{gr} / \mathrm{dm}^{3}$ for loam. The difference is in good agreement with moisture determinations based on the calibration curves.

This agreement is far less satisfactory when one compares sand and loam. This may be due to a lack of precision in the lower, less steep parts of the calibration curves.

3 Following a procedure indicated by BRocand (1954), we made a second series of experiments, protecting the indium detector by two coaxial cadmium cylinders. Indium has a resonance peak for capture of neutrons of $1.4 \mathrm{eV}$. As the cross section for capture of thermal neutrons is very high for cadmium, the detector is mainly activated by resonance neutrons of $1.4 \mathrm{eV}$. As the detected neutrons are reasonably monoenergetic, an increase of accuracy can be expected. The corresponding calibration curves (Fig. 2) show accordingly an improved precision in the region of low moisture contents. 


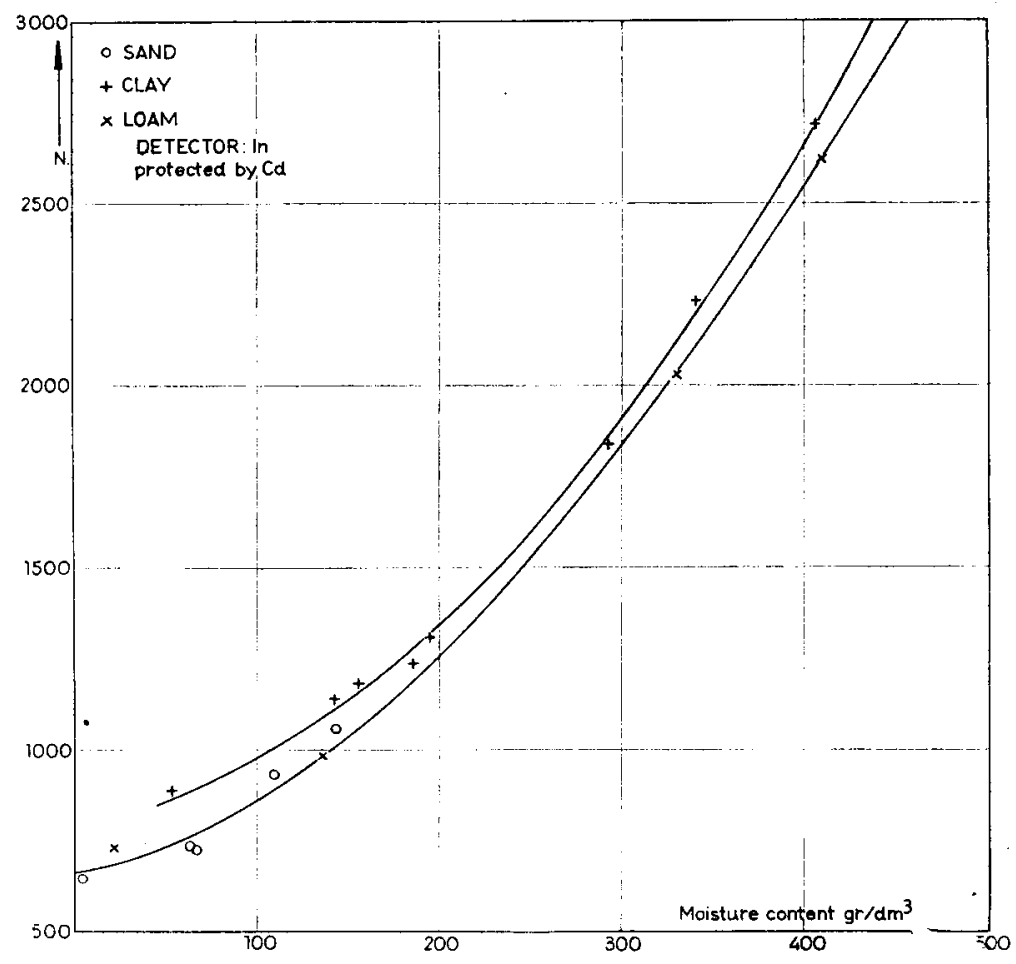

Fig. 2. Counting rate vs. moisture content for several solls.

This investigation was performed under the auspices of the Nationaal Fonds voor Wetenschappelijk Onderzoek, Brussels.

\section{REFERENCES}

Gardner, W. and D. Kirkham: Soil Sci. 73 (1952) 391.

Belcher, D. J., T. R. Cuykendalt and H. A. SACK : Technical Development Report, U.S. Civil Aeronautics Administration, nrs. 127 (1950), 194 (1953).

De LeEnheEr, L. : Mededelingen Rijkslandbouwhogeschool, Gent, IX (1941) 121.

Brocard, J.: R.I.L.E.M., Dec. 1954. 